\title{
AUTOMATIC CHOICE OF QUADRATURE ORDER FOR EFFICIENT EVALUATION OF SURFACE VIEW FACTORS
}

\author{
R.P. Koptelov, Postgraduate \\ A.M. Konashkova, Cand.Eng., Assist. professor \\ Ural Federal University (Ekaterinburg, Russia) \\ E-MAIL:r-koptelov@mail.ru
}

Abstract. View factors for two real geometries of continuous furnace with cylindrical bars and sectional pusher furnace of ball-rolling workshop are calculated with the help of previously developed method of automatic choosing the number of quadrature nodes. It is shown that the method is several times faster and also more accurate than standard calculation.

Keywords: radiative heat transfer, view factors, integration.

\section{REFERENCES}

1. Mod e s t M.F. Radiative Heat Transfer. Second Edition. Academic Press. 2003.

2. L i s i e n k o V.G. Sovershenstvovanie i povyshenie jeffektivnosti jenergotehnologij i proizvodstv. Integrirovannyj jenergo-jekologicheskij analiz: teorija i praktika. T. 1 (Improved and effective energy technologies and industries. Integrated Energy and Environmental Analysis: Theory and Practice. Vol. 1). Moscow: Teplotehnik, 2010. 688 p.

3. Emery A.F., Johansson O., Lobo M., Abrous A.A Comparative Study of Methods for Computing the Diffuse Radiation Viewfactors for Complex Structures». ASME Journal of Heat Transfer. 1991. Vol. 113. pp. 413 - 422.

4. Malikov G.K., Lis i enko V.G., Koptelov R.P. Izv. vuz. Chernaja metallurgija. 2010. № 7. pp. 53 - 59.

5. Koptelov R.P., Malikov G.K., Lis i enko V.G. Izv. vuz. Chernaja metallurgija. 2013. № 1. pp. 23 - 31.

6. Ravishankar M., Mazumder S. Application of the Modifed Differential Approximation (MDA) for Radiative Transport to arbitrary Three-Dimensional Geometry. Proceedings of the IMECE. 2009. Paper Number - ICEME2009-12844.

7. Walt on G.N. Calculation of obstructed view factors by adaptive integration. National Institute of Standards and Technology. NISTIR 6925. 2002.

8. Mazumder S. Methods to accelerate ray tracing in the Monte Carlo method for surface-to-surface radiation transport. ASME Journal of Heat Transfer. 2006. Vol. 128. No 9. pp. 945 - 952.

Received 3 September 2013

УДК 669.041:621.783:66.045

\section{О ПРОИЗВОДИТЕЛЬНОСТИ НАГРЕВАТЕЛЬНЫХ ПЕЧЕЙ С МИНИМАЛЬНЫМ РАСХОДОМ ТОПЛИВА}

\author{
А.К. Соколов, д.т.н., профессор
}

Ивановский государственный энергетический университет (Иваново, Россия)

\begin{abstract}
Аннотация. Показана актуальность исследований производительности печей, обеспечивающих минимальный удельный расход топлива. Пред-

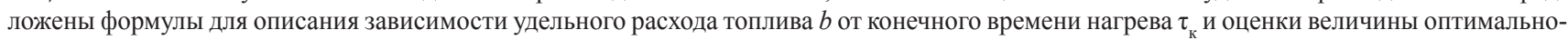
го по расходу топлива конечного времени нагрева $\tau_{\text {ко }}$ (производительности). Приведены результаты исследования зависимостей $b\left(\tau_{\kappa}\right)$ и $\tau_{\text {ко }}$ от удельных потерь теплоты теплопередачей из рабочего пространства печей и способа отвода продуктов сгорания. Установлено, что значение оптимальной производительности в значительной степени определяется величиной потерь теплоты теплопередачей, которые обратно пропорционально влияют на оптимальное значение производительности. Показаны примерные условия, при которых минимальный удельный расход топлива может наблюдаться как при предельно высокой производительности, так и при низкой производительности печи.

Ключевые слова: газовые печи, нагрев металла, производительность, оптимальный режим.
\end{abstract}

\section{E-MAIL: sokolov@bjd.ispu.ru}

Можно считать общепринятым фактом наличие зависимости удельного расхода топлива $b$ на нагрев металла от заданного конечного времени нагрева $\tau_{\mathrm{\kappa}}$ или производительности $P$, которые связаны соотношением $P=M / \tau_{\text {к }}$, где $M-$ масса садки металла, кг.

Однако в литературе пока еще недостаточно четко определены ответы на следующие важные для теории и практики вопросы.

- Имеется ли минимум у зависимости $b\left(\tau_{\kappa}\right)$ в практически важной области изменения производительности?

- Находится ли зона минимума $b\left(\tau_{\kappa}\right)$ вблизи минимально допустимого (наискорейшего) для данных ус- ловий времени нагрева $\tau_{\text {кн }}$ (максимальной производительности)?

- Какие характеристики печи в основном определяют значение $\tau_{\text {ко }}$, при котором удельный расход топлива минимален и знак производной $d b / d \tau_{\mathrm{\kappa}}$ при $\tau_{\mathrm{K}}>\tau_{\text {кн }}$. Отметим, что при $d b / d \tau_{\mathrm{K}}<0$ увеличение $\tau_{\mathrm{K}}$ (снижение производительности) будет приводить к уменьшению удельного расхода топлива.

В работе [1] сделан обзор публикаций, в которых высказаны мнения по некоторым из названных проблем. В большей части из них утверждается, что минимальный удельный расход топлива на нагрев металла требуется при наибольшей производительности (или 
при $\left.\tau_{\kappa}=\tau_{\text {кн }}\right)$. В данном обзоре [1] дана ссылка лишь на одну работу, в которой указывается, что «с ростом производительности увеличивается удельный расход топлива на нагрев, однако такой режим имеет место при очень малых потерях теплоты (до 2 \% общей тепловой мощности печи)».

В работах автора по исследованию режимов нагрева металла с минимальным расходом топлива не были обнаружены условия, при которых минимальный расход топлива, а также минимальный угар металла наблюдался бы при наибольшей производительности [3 - 5].

Естественно, что решение о величине оптимальной производительности не должно приниматься большинством голосов. Ответ на этот вопрос не может быть однозначным и должен зависеть от конкретных условий работы нагревательной печи. Для поиска ответов на вопросы, сформулированные в начале статьи, необходимы более широкие теоретические исследования.

Численные методы расчета, которые используются при моделировании режимов нагрева металла в печах, позволяют получить достаточно надежные значения оптимального времени нагрева $\tau_{\text {ко }}$. Недостаток их применения заключается в трудности обобщения результатов моделирования работы печей при различных условиях.

Аналитические модели, которые на современном уровне развития металлургической теплотехники используются лишь для качественной оценки техникоэкономических показателей работы печей, дают возможность более широкого обобщения результатов.

Рассмотрим методику аналитического исследования зависимости $b\left(\tau_{\mathrm{\kappa}}\right)$.

Для оценки оптимального по расходу топлива времени нагрева $\tau_{\text {ко }}$ необходимо найти аналитические зависимости удельного расхода топлива $b$ от конечного времени нагрева $\tau_{\kappa}$ и условий работы печи.

Запишем уравнение баланса теплоты рабочего пространства печи, в которой нагревается садка металла массой $M$, кг:

$$
B\left(i_{\mathrm{T}}-i_{\mathrm{yx}}\right)=I_{\mathrm{M}}+I_{\Pi},
$$

где $B$ - объем сжигаемого топлива, $\mathrm{M}^{3}$ (здесь и далее объемы газов даны при нормальных условиях); $i_{\text {т }}$ - энтальпия продуктов сгорания, учитывающая химическую и физическую теплоту топлива и воздуха на горение, Дж/м ${ }^{3}$ топлива; $i_{\text {ух }}$ - энтальпия уходящих газов, Дж/м ${ }^{3}$ топлива; $I_{\text {м }}$ - количество теплоты, затраченной на нагрев садки металла, Дж; $I_{п}$ - количество теплоты, теряемой из рабочего пространства через кладку (ограждение), окна и в водоохлаждаемые элементы печи, Дж.

В таком виде уравнение баланса справедливо для проходных (методических), секционных печей и печей периодического действия.
Разделим обе части уравнения (1) на массу садки $M$ и выразим из него удельный расход топлива $b=B / M$, $\mathrm{M}^{3} / \mathrm{\kappa} \Gamma$ :

$$
b=\frac{\frac{I_{\mathrm{M}}+I_{\mathrm{I}}}{M}}{i_{\mathrm{T}}-i_{\mathrm{yx}}} .
$$

Величины $I_{\text {м }}, i_{\text {т }}$ и $M$ не зависят от времени нагрева садки металла. На динамику изменения функции $b\left(\tau_{\kappa}\right)$ влияют только величины $I_{\text {п }}$ и $i_{\mathrm{yx}}$. Представим эти параметры функциями времени нагрева.

Потери теплоты выразим через средний поток теплоты, теряемой теплопередачей $Q_{\text {п }}$ :

$$
I_{\Pi}=Q_{\Pi} \tau_{\kappa}
$$

где $Q_{\text {п }}=q_{\text {п }} F \omega, \mathrm{Bт}, q_{\text {п }}-$ средний удельный поток теплоты, Вт/м² кладки; $F$ - площадь обогреваемой поверхности металла, м $^{2} ; \omega$ - степень развития обмуровки, $\omega=F_{\text {кл }} / F$, $F_{\text {кл }}$ - площадь внутренней поверхности кладки, ${ }^{2}$. При наличии потерь теплоты через окна, водоохлаждаемые элементы, величины $q_{\text {п }}, F_{\text {кл }}, \omega$ должны быть приведенными к условиям теплопередачи через кладку.

Энтальпия уходящих газов зависит от температуры уходящих газов $T_{\mathrm{y}}$ :

$$
i_{\mathrm{yx}}=c v T_{\mathrm{y}},
$$

где $c$ - теплоемкость продуктов сгорания, Дж/ $/ \mathrm{M}^{3} \cdot \mathrm{K}$; $v$ - удельный объем продуктов сгорания $1 \mathrm{M}^{3}$ топлива, $\mathrm{M}^{3} / \mathrm{M}^{3}$.

В общем случае величины $q_{\text {п }}$ и $c$ зависят от температуры газов (режима нагрева) и, следовательно, от времени нагрева. Примем, что эти зависимости для качественного (приближенного) анализа несущественны.

Наибольшие проблемы возникают при описании зависимости температуры уходящих газов $T_{\text {у }}$ от конечного времени нагрева, которая должна снижаться при увеличении $\tau_{\kappa}$. Рассматривалось несколько вариантов выражения функции $T_{\mathrm{y}}\left(\tau_{\mathrm{\kappa}}\right)$. Был принят весьма приближенный способ выражения $T_{\text {у через }}$ средний перепад температур между газом и металлом $\Delta T_{\Gamma-\mathrm{M}}=\mathrm{I}_{\mathrm{M}} /\left(\tau_{\mathrm{\kappa}} \alpha F\right)$ :

$$
T_{\text {у }}=T_{\text {му }}+\frac{I_{\text {м }}}{\tau_{\text {к }} \alpha F},
$$

где $\alpha$ - приведенный коэффициент теплоотдачи от газов к металлу, Вт/( $\left.{ }^{2} \cdot \mathrm{K}\right) ; T_{\text {му }}$ с среднеинтегральная температура поверхности металла за время нагрева $\tau_{\mathrm{\kappa}}$ для секционных печей и печей периодического действия или температура поверхности металла в районе отвода продуктов сгорания (начало методической зоны).

Выразим зависимость $b\left(\tau_{\kappa}\right)$, подставив формулы (3) - (5) в (2): 


$$
b=\frac{\frac{I_{\mathrm{M}}+Q_{\mathrm{\Pi}} \tau_{\mathrm{K}}}{M}}{i_{\mathrm{T}}-c \mathrm{v}\left(\frac{I_{\mathrm{M}}}{\tau_{\mathrm{K}} \alpha F}+T_{\mathrm{My}}\right)} .
$$

Используя выражение (6), можно классическим методом определить время $\tau_{\text {ко }}$, при котором расход топлива будет минимален. Возьмем производную выражения (6) по $\tau_{\kappa}$ и приравняем ее нулю:

$$
\begin{aligned}
& \frac{d b}{d \tau_{k}}\left(\frac{\frac{I_{\mathrm{M}}}{M}+Q_{\mathrm{I}} \frac{\tau_{\mathrm{K}}}{M}}{i_{\mathrm{T}}-c \mathrm{v}\left[\frac{I_{\mathrm{M}}}{\tau_{\mathrm{K}} \alpha F}+T_{\mathrm{My}}\right]}\right)= \\
& =\frac{\frac{I_{\mathrm{M}}}{M}}{-\left\{i_{\mathrm{T}}-c \mathrm{v}\left[\frac{I_{\mathrm{M}}}{\tau_{\mathrm{K}} \alpha F}+T_{\mathrm{My}}\right]\right\}^{2}} \frac{\left(-\frac{c \cup I_{\mathrm{M}}}{\alpha F}\right)}{\left(-\tau_{\mathrm{K}}^{2}\right)}+ \\
& +\frac{\frac{Q_{\mathrm{II}}}{M}}{i_{\mathrm{T}}-c \mathrm{v}\left[\frac{I_{\mathrm{M}}}{\tau_{\mathrm{K}} \alpha F}+T_{\mathrm{My}}\right]}+\frac{\frac{Q_{\mathrm{II}} \tau_{\mathrm{K}}}{M}}{-\left\{i_{\mathrm{T}}-c \mathrm{v}\left[\frac{I_{\mathrm{M}}}{\tau_{\mathrm{K}} \alpha F}+T_{\mathrm{My}}\right]\right\}^{2}} \times \\
& \times \frac{\left(-\frac{c \cup I_{\mathrm{M}}}{\alpha F}\right)}{\left(-\tau_{\mathrm{K}}^{2}\right)}=0 .
\end{aligned}
$$

Приведем слагаемые к одному знаменателю, умножим его на нуль правой части равенства (7) и получим:

$$
\begin{gathered}
-\frac{I_{\mathrm{M}}^{2} c \mathrm{v}}{\alpha F M}+\frac{Q_{\mathrm{\Pi}}}{M} \tau_{\mathrm{\kappa}}^{2}\left\{i_{\mathrm{T}}-c \mathrm{v}\left[\frac{I_{\mathrm{M}}}{\tau_{\mathrm{K}} \alpha F}+T_{\mathrm{My}}\right]\right\}- \\
-Q_{\mathrm{\Pi}} \tau_{\mathrm{K}} c v \frac{I_{\mathrm{M}}}{\alpha F M}=0 .
\end{gathered}
$$

Умножим (8) на $\frac{\alpha F M}{Q_{\text {п }} c \cup I_{\text {м }}}$ и преобразуем его к виду квадратного уравнения

$$
\frac{\alpha F}{I_{\mathrm{M}}}\left(\frac{i_{T}}{c \mathrm{v}}-T_{\mathrm{My}}\right) \tau_{\mathrm{\kappa}}^{2}-2 \tau_{\mathrm{\kappa}}-\frac{I_{\mathrm{M}}}{Q_{\mathrm{m}}}=0 .
$$

Оценить значение $\tau_{\text {ко }}$ можно по решению квадратного уравнения (9), которое имеет вид

$$
\tau_{\text {ко }}=\frac{2+\sqrt{4+4 \alpha F\left(\frac{\frac{i_{\mathrm{T}}}{c \mathrm{v}}-T_{\mathrm{My}}}{Q_{\mathrm{n}}}\right)}}{2 \alpha F\left(\frac{\frac{i_{\mathrm{T}}}{c \mathrm{v}}-T_{\mathrm{My}}}{I_{\mathrm{M}}}\right)}
$$

или

$$
\tau_{\mathrm{ko}}=\frac{\left.1+\sqrt{1+\alpha F\left(\frac{\frac{i_{\mathrm{T}}}{c \mathrm{v}}-T_{\mathrm{My}}}{Q_{\mathrm{\Pi}}}\right)}\right)}{\alpha F\left(\frac{\frac{i_{\mathrm{T}}}{c \mathrm{v}}-T_{\mathrm{My}}}{I_{\mathrm{M}}}\right)} .
$$

Анализ выражения (10) позволяет определить характер изменения параметров, которые будут сокращать оптимальное время нагрева (увеличивать оптимальное значение производительности):

- увеличение потерь теплоты теплопередачей;

- снижение калориметрической температуры сгорания топлива $T_{\text {кал }}=i_{\mathrm{T}} /(c \mathrm{v})$;

- увеличение интенсивности теплоотдачи к металлу $\alpha$;

- снижение температуры уходящих газов за счет организации их отвода из низкотемпературной (методической) зоны и уменьшения $T_{\text {му }}$ [уравнения (5), (10)].

По уравнению (9) с учетом (3) можно оценить величину средних удельных потерь теплоты $q_{\text {по }}$, при которой расход топлива для заданного конечного времени

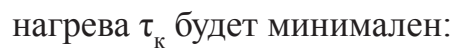

$$
q_{\text {по }}=\frac{I_{\mathrm{M}}^{2}}{\left[\alpha F \tau_{\mathrm{\kappa}}^{2}\left(\frac{i_{\mathrm{T}}}{c \nu}-T_{\mathrm{му}}\right)-2 I_{\mathrm{M}} \tau_{\kappa}\right] F \omega} .
$$

Если в уравнении (11) принять $\tau_{\mathrm{K}}=\tau_{\text {кн }}$, то можно оценить, при каких потерях теплоты наискорейшее время нагрева обеспечит минимальный удельный расход топлива на нагрев металла.

Для исследования влияния производительности (времени нагрева) на удельный расход топлива выполним расчет одностороннего нагрева металла в форме неограниченной пластины при следующих исходных данных: теплоемкость $c_{\mathrm{M}}=650$ Дж/кг $\cdot$ К; плотность $\rho=7850$ кг $/ \mathrm{M}^{3}$; толщина металла $R=0,1$ м; среднемассовые конечные и начальные температуры металла $T_{\mathrm{K}}=1227^{\circ} \mathrm{C}(1500 \mathrm{~K})$; $T_{\mathrm{H}}=27^{\circ} \mathrm{C}(300 \mathrm{~K}) ; \alpha=220 \mathrm{BT} / \mathrm{m}^{2} \cdot \mathrm{K} ; F=30 \mathrm{~m}^{2} ; \omega=3$;

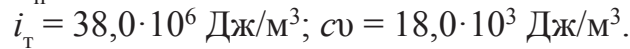

Определим количество теплоты, затраченной на нагрев металла и массу садки, Дж:

$$
\begin{gathered}
I_{\mathrm{M}}=c_{\mathrm{M}} \rho R\left(T_{\mathrm{\kappa}}-T_{\mathrm{H}}\right) . \\
I_{\mathrm{M}}=650 \cdot 7850 \cdot 0,1 \cdot(1227-27)=184 \cdot 10^{8} \text { Дж; } \\
M=\rho R F=7850 \cdot 0,1 \cdot 30=23550 \text { кг. }
\end{gathered}
$$

Исходные показатели приняты близкими к данным, которые использовались в работе [3], чтобы оценивать значение $\tau_{\text {кн }}$, сравнивая результаты расчетов.

На рис. 1 приведены результаты расчетов $b$ и $T_{\text {y }}$ по формулам (6) и (5) для $R=0,1$ и 0,2 м при за- 
данных удельных потерях теплоты теплопередачей $q_{\text {п }}=2 \cdot 10^{3} ; 12 \cdot 10^{3} ; 22 \cdot 10^{3} \mathrm{BT} / \mathrm{M}^{2}\left(Q_{\text {п }}=0,18 ; 1,08\right.$; $1,98 \mathrm{MB}$ ) и $T_{\text {му }}=650{ }^{\circ} \mathrm{C}$. Такое значение $T_{\text {му }}$ примерно определит по уравнению (5) величины температур уходящих газов для печей с отводом продуктов сгорания из каждой зоны или средних за периоды нагрева температур $T_{\mathrm{y}}$.

Графики изменения удельного расхода топлива показывают, что функции $b\left(\tau_{\mathrm{\kappa}}\right)$ имеют минимумы, а значение $\tau_{\text {ко }}$, при котором $b\left(\tau_{\kappa}\right)=\min$, при увеличении

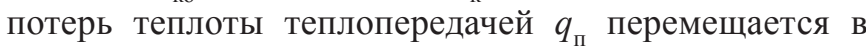
сторону более высоких оптимальных производительностей печей.

Обратим внимание, что для этих печей даже при сравнительно высоких потерях теплоты (в данных условиях) оптимальная производительность не является предельной $\left(\tau_{\text {ко }}>\tau_{\text {кн }}\right)$.

И только при $R=0,2$ и $q_{\text {п }}=22$ кВт $\left(Q_{\text {п }}=1,98\right.$ МВт $)$ оптимальная производительность находится в зоне предельных значений.

На рис. 2 приведены аналогичные зависимости для проходных печей с отводом всех газов из одной «холодной» (методической) зоны $\left(T_{\text {му }}=300{ }^{\circ} \mathrm{C}\right)$.

При более низком значении $T_{\text {му }}$ и, соответственно, $T_{\text {y }}$, значения $\tau_{\text {ко }}$ более, чем при $T_{\text {му }}^{\text {му }}=650{ }^{\circ} \mathrm{C}$, смещаются ближе к $\tau_{\text {кн. }}$. Оптимальная производительность находится в зоне предельной при $q_{\text {п }}>22$ кВт/м ${ }^{2}(R=0,1$ м) и $q_{\text {п }} \geq 12$ кВт/м ${ }^{2}(R=0,2 \mathrm{м})$. Величина $b$ в зоне оптимума $\tau_{\mathrm{\kappa}} \approx \tau_{\text {ко }}$ довольно слабо зависит от производительности. Причем возможны такие условия, когда величина $b$ довольно слабо зависит от выбранной производительности в большом диапазоне изменения $\tau_{\mathrm{\kappa}}\left(q_{\text {п }}=12 \mathrm{\kappa BT} / \mathrm{M}^{2}\right.$, $R=0,2 \mathrm{M})$.

Обратим внимание, что эффект оптимизации (возможность снижения удельного расхода топлива за счет выбора производительности) на рис. 1 и 2 оказался меньше, чем в более точных расчетах [3] из-за приближенного учета $q_{\text {п }}$ и зависимости $T_{\mathrm{y}}\left(\tau_{\mathrm{\kappa}}\right)$ в виде (5).

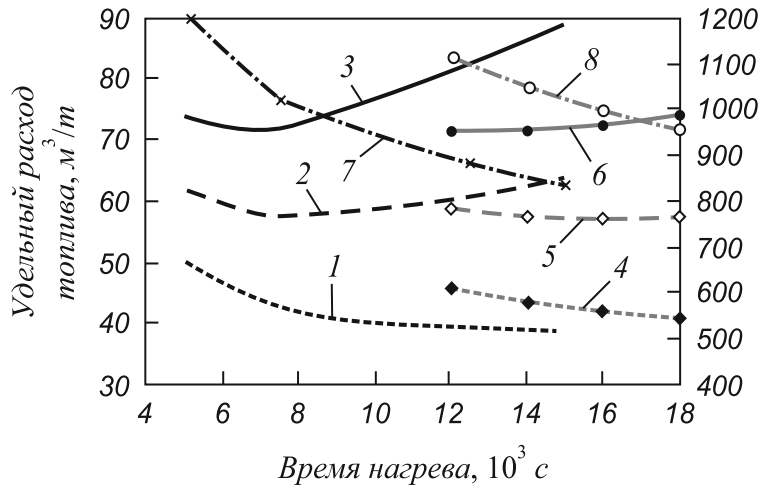

Рис. 1. Удельный расход топлива и температура уходящих газов в зависимости от конечного времени нагрева при нагреве металла толщиной $R=0,1$ и 0,2 м с удельными потерями теплоты $q_{\text {п }}=2,12$

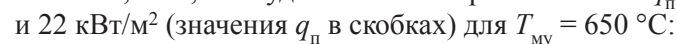

$1-R=0,1$ (2); $2-R=0,1$ (12); $3-R=0,1$ (22); $4-R=0,2$ (2);

$5-R=0,2(12) ; 6-R=0,2(22) ; 7-T_{\Gamma}, R=0,1 ; 8-T_{\Gamma}, R=0,2$
Естественно, что представленные зависимости $b\left(\tau_{\mathrm{\kappa}}, q_{\text {п }}\right)$ были бы более информативны, если бы вместо $q_{\text {п }}$ и $Q_{\text {п }}$ использовать их относительные величины:

$$
D_{\mathrm{p} / \mathrm{M}}=Q_{\Pi} \frac{\tau_{\mathrm{\kappa}}}{I_{\mathrm{M}}}
$$

или

$$
D_{\mathrm{p}}=Q_{\Pi} \frac{\tau_{\mathrm{\kappa}}}{b M i_{\mathrm{T}}} .
$$

Однако это оказалось невозможно, так как $D_{\mathrm{p} / \mathrm{m}}$ и $D_{\mathrm{p}}$ зависят от $\tau_{\kappa}$.

В таблице приведены значения относительных потерь теплоты $D_{\mathrm{p}}$, рассчитанные по формуле (13) для различных $\tau_{\text {к }}$ и $R=0,1 \mathrm{M}, T_{\text {му }}=300$ (см. рис. 2).

Как следует из данных таблицы, величина $D_{\mathrm{p}}$ изменяется в широких пределах (от 3 до 48 \%). Для данных условий сокращение времени нагрева (увеличение производительности) увеличивает $b$ при доле потерь меньше $\sim 14 \%\left(d b / d \tau_{\text {к }}<0\right)$. Увеличение времени нагрева (уменьшение производительности) увеличивает $b$ при доле потерь больше 20\%.

Этот и другие выводы, касающиеся граничных значений параметров, влияющих на $\tau_{\text {ко }}$, весьма приближенны и приводятся только для доказательства того, что величина оптимальной производительности существенно зависит от конструкции и условий работы печи.

В качестве примера и доказательства правильности вывода формулы (10) оценим оптимальное время нагрева при $R=0,1 \mathrm{м}, I_{\mathrm{м}}=184 \cdot 10^{8}$ Дж, $q_{\text {п }}=2 \cdot 10^{3} \mathrm{B \textrm {T }} / \mathrm{M}^{2}$, $Q_{\text {п }}=1,08 \cdot 10^{6}$ Вт и $T_{\text {му }}=650^{\circ} \mathrm{C}$ :
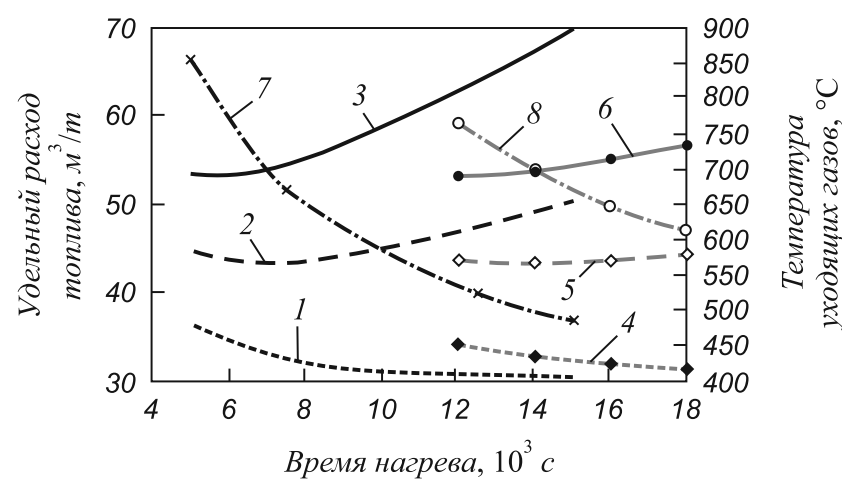

Рис. 2. Удельный расход топлива и температура уходящих газов в зависимости от конечного времени нагрева для $T_{\text {му }}=300{ }^{\circ} \mathrm{C}$ (обозначения см. рис. 1)

Относительные потери теплоты теплопередачей $D_{\mathrm{p}}$ (числитель) и удельные расходы топлива $b$ (знаменатель) при $R=0,1 \mathrm{M}, T_{\text {му }}=300 \mathrm{~K}$

\begin{tabular}{c|c|c|c|c}
\hline \multirow{2}{*}{$q_{\text {п }}, \mathrm{\kappa BT} / \mathrm{M}^{2}$} & \multicolumn{4}{|c}{$\tau_{\mathrm{\kappa}}, \mathrm{c}$} \\
\cline { 2 - 5 } & 5000 & 7500 & 12500 & 15000 \\
\hline 2 & $2,8 / 36,24$ & $4,7 / 32,30$ & $8,2 / 30,62$ & $9,9 / 30,58$ \\
\hline 12 & $13,5 / 44,70$ & $20,9 / 43,36$ & $31,9 / 47,33$ & $36,1 / 50,17$ \\
\hline 22 & $20,8 / 53,16$ & $30,5 / 54,42$ & $43,2 / 64,04$ & $47,6 / 69,76$ \\
\hline
\end{tabular}




$$
\tau_{\text {ко }}=\frac{1+\sqrt{1+220 \cdot 30 \cdot \frac{\frac{38 \cdot 10^{6}}{18 \cdot 10^{3}}-650}{1,08 \cdot 10^{6}}}}{220 \cdot 30 \cdot \frac{\frac{38 \cdot 10^{6}}{18 \cdot 10^{3}}-650}{184 \cdot 10^{8}}}=7920 \mathrm{c} .
$$

Значение $\tau_{\text {ко }}=7920$ с соответствует минимуму функции $b\left(\tau_{\mathrm{K}}\right)$ на рис. 1.

Оценим величину удельных $q_{\text {по }}$ и относительных потерь теплоты $D_{\text {ро }}$, при которых минимальный удельный расход топлива на нагрев металла будет затрачиваться при наискорейшем времени нагрева $\left(\tau_{\mathrm{\kappa}}=\tau_{\mathrm{\kappa H}}\right)$ для $R=0,2 \mathrm{M} ; \tau_{\text {кн }} \approx \tau_{\mathrm{K}}=12000 \mathrm{c} ; I_{\mathrm{M}}=650 \cdot 7850 \cdot 0,3 \cdot(1227-27)=$ $=368 \cdot 10^{8}$ Дж; $M=7850 \cdot 0,2 \cdot 30=47100$ кг; $T_{\text {му }}=300{ }^{\circ} \mathrm{C}$. Остальные параметры такие же, как в предыдущем примере.

Величина $q_{\text {по }}$ по формуле (11) составит

$$
\begin{gathered}
q_{\text {по }}=\left(368 \cdot 10^{8}\right)^{2} /\left\{\left[220 \cdot 30 \cdot 12000^{2}\left(\left(\frac{38 \cdot 10^{6}}{18 \cdot 10^{3}}\right)-300\right)-\right.\right. \\
\left.\left.-2 \cdot 368 \cdot 10^{8} \cdot 12000\right] \cdot 30 \cdot 3\right\}=17950 .
\end{gathered}
$$

Значение $q_{\text {по }}=17950 \mathrm{BT} / \mathrm{M}^{2}$ соответствует положению минимума функции $b\left(\tau_{\mathrm{\kappa}}\right)$ на рис. 2 .

Рассчитаем удельный расход топлива по формуле (6) с учетом (3):

$$
\begin{gathered}
b=\frac{\frac{368 \cdot 10^{8}+17950 \cdot 30 \cdot 3 \cdot 12000}{47100}}{38 \cdot 10^{6}-18 \cdot 10^{3} \cdot\left(\frac{368 \cdot 10^{8}}{12000 \cdot 220 \cdot 30}+300\right)}= \\
=0,049 \mathrm{M}^{3} / \mathrm{K} \Gamma=49 \mathrm{M}^{3} / \mathrm{T}
\end{gathered}
$$

и оценим относительную величину потерь теплоты по формуле (13):

$$
\begin{gathered}
D_{\mathrm{p}}=17950 \cdot 30 \cdot 3 \cdot 1200 /\left(0,049 \cdot 47100 \cdot 38 \cdot 10^{6}\right)= \\
=0,22=22 \% .
\end{gathered}
$$

Таким образом, в данном случае при относительных потерях теплоты теплопередачей $D_{\mathrm{p}}=22 \%$, работа при производительности, близкой к предельной (наискорейшем времени $\tau_{\text {кн }} \approx 12000 \mathrm{c}$ ), потребует минимального удельного расхода топлива. При снижении потерь теплоты в 4 раза до $q_{\text {п }}=17950 / 4=4488 \mathrm{BT} / \mathrm{M}^{2}$ значение оптимального времени возрастет от $12000 \mathrm{c}$ до $\tau_{\text {ко }}=20100$ с (более чем на 2 ч или более чем в полтора раза).

Для оценки степени влияния температуры уходящих газов (или $T_{\text {му }}$ ) и потерь теплоты на рис. 3 приведены

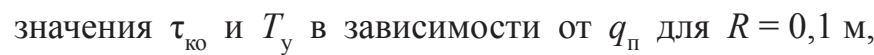
$T_{\text {му }}=100,500,900^{\circ} \mathrm{C}$. Значение $\tau_{\text {кн }}$ для данных условий составляет около 5000 с. Из рис. 3 видно, что увеличение удельных потерь теплоты от 2 до 14 кВт/м² существенно влияет на значения $\tau_{\text {ко }}$, а затем это влияние становится слабее.

Поскольку величина $q_{\text {п }}$ существенно влияет на значения $\tau_{\text {ко }}$, были выведены формулы для расчета $b\left(\tau_{\mathrm{k}}\right)$ при учете зависимости удельных потерь теплоты от средней температуры газов в печи $T_{\Gamma}$ по формуле

$$
q_{\text {п }}=\frac{a_{q}+b_{q} T_{\mathrm{r}}}{1+\frac{b_{q}}{\alpha_{\text {гк }}}},
$$

где $a_{q}, b_{q}$ - коэффициенты аппроксимации зависимости $q_{\text {п }}=a_{q}+b_{q} T_{\text {кл }}[6] ; \alpha_{\text {гк }}-$ приведенный коэффициент конвективной теплоотдачи от газа к кладке; $T_{\text {кл }}$ средняя температура внутренней поверхности кладки печи.

Расчеты показали, что использование зависимости (14) принципиально не изменило результаты, приведенные на рис. 1 - 3 .

На основании изложенного можно полагать, что величина оптимального времени существенно зависит от условий нагрева, поэтому обобщающие утверждения, такие как наискорейший нагрев является оптимальным, некорректны. Свидетельствовать о величине оптимальной производительности можно только для конкретной печи и конкретных условий ее работы (свойства металла, схема отвода продуктов сгорания, потери теплоты теплопередачей, состояние тепловой изоляции и др.)

Для определения более достоверных значений $\tau_{\text {ко }}$ следует использовать более сложные математические модели или результаты промышленных испытаний или статистические данные о работе печи. Приведенные выше приближенные формулы можно применять лишь для качественной оценки взаимосвязей парамет-

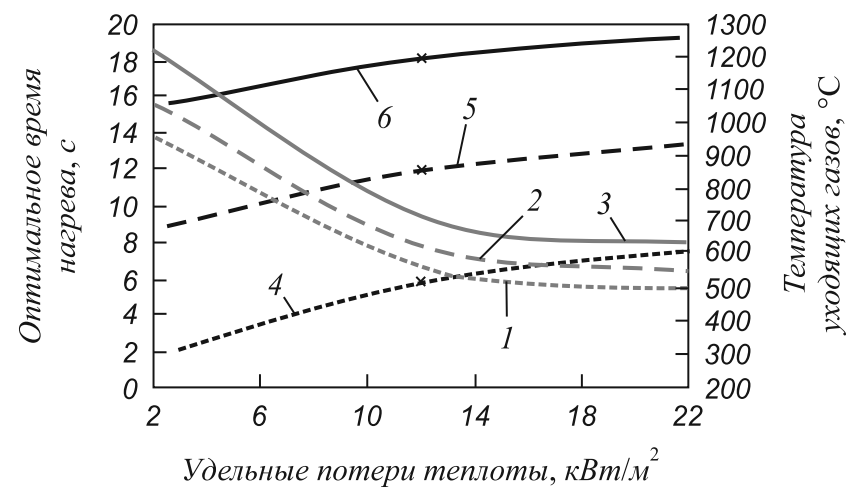

Рис. 3. Оптимальное по удельному расходу топлива время нагрева $\tau_{\text {ко }}$ и температура уходящих газов $T_{\Gamma}$ в зависимости от удельных потерь теплоты для $R=0,1$ м и различных значений $T_{\text {му }}=100,500,900{ }^{\circ} \mathrm{C}$ (значения в скобках):

$1-\tau_{\text {ко }}(100) ; 2-\tau_{\text {ко }}(500) ; 3-\tau_{\text {ко }}(900) ; 4-T_{\text {г }}(100) ; 5-T_{\text {г }}(500)$; $6-T_{\Gamma}(900)$ 
ров печей. Их целесообразно использовать также в учебном процессе при изучении простейшей математической модели тепловой работы печи и оценки степени влияния параметров на удельный расход топлива. Проведение исследований по формулам (1) - (13) будет достаточно наглядным и потребует простейших программных средств.

Bыводы. Предложены простые формулы для оценки оптимального по удельному расходу топлива конечного времени нагрева (производительности). Исследовано влияние удельных потерь теплоты теплопередачей и способа отвода продуктов сгорания на величину оптимальной производительности. Установлено, что значение оптимальной производительности в значительной степени определяется величиной потерь теплоты теплопередачей, поэтому ухудшение тепловой изоляции ограждений, водоохлаждаемых элементов изменяет (увеличивает) оптимальное значение производительности.

Показаны условия, при которых предельно высокая производительность может быть оптимальной и вероятность условий, при которых производительность будет слабо влиять на величину удельного расхода топлива (см. рис. 1 и 2).

Эффект оптимизации производительности снижается при увеличении определяющего размера (толщины) металла, а размер зоны оптимума, в пределах которой величина $b$ изменяется в пределах $1 \%$, может быть достаточно большим $2000-4000$ с.

Наконец, главный вывод (предложение) при описании каких-либо «закономерностей», «случаев» или ссылке на них заключается в том, что следует избегать обобщающих заключений и, по возможности, четко указывать область параметров, в которых обнаруженные закономерности и принципы соблюдаются, чтобы не вводить в заблуждение практиков.

\section{БИБЛИОГРАФИЧЕСКИЙ СПИСОК}

1. П а н фе р о в В.И. // Изв. вуз. Черная металлургия. 2007. № 10. C. $53-56$.

2. Теплообмен и тепловые режимы в промышленных печах / Ю.И. Розенгарт, Б.Б. Потапов, В.М. Ольшанский, А.В. Бородулин. - Киев -Донецк: Вища школа, 1986. - 296 с.

3. С окол о в А.К.// Изв. АН. Энергетика. 2007. № 2. С. 126 - 136.

4. Ревун М.П., Соколов А.К. Адаптивные системы управления процессами нагрева металла. - Запорожье: Изд-во ЗГИА, 1998. $-351 \mathrm{c}$.

5. С околов А.К. Совершенствование и оптимизация нагрева металла в газовых печах методом математического моделирования. - Иваново: ИГЭУ, 2012. - 256 с.

6. С окол о в А.К.// Изв. вуз. Энергетика. 1981. № 5. С. $104-106$.

(C) 2014 г. А.К. Соколов Поступила 8 октября 2013 г.

\section{ON THE PRODUCTION OF HEATING FURNACES WITH MINIMUM FUEL CONSUMPTION}

\section{A.K. Sokolov, Dr.Eng., Professor}

Ivanovo State Energy University (Ivanovo, Russia)

E-MAIL: sokolov@bjd.ispu.ru

Abstract. The article shows the urgency of research of furnaces productivity of the furnaces, that provide a minimum specific fuel consumption $b$. Proposed a relatively simple formula to describe the dependence $b\left(\tau_{\mathrm{K}}\right)$ of particular fuel consumption of the final $b$ heating time $\tau_{\mathrm{K}}$, and estimate the optimal fuel consumption of the final heating time $\tau_{\mathrm{KO}}$ (performance). Results on $b\left(\tau_{\mathrm{K}}\right)$ and $\tau_{\mathrm{Ko}}$ for different specific heat loss heat transfer from the volume $q_{\Pi}$ furnaces and flue gas ways are described. It has been established that the optimum value of the performance is largely determined of heat losses by the heat transfer, which are inversely proportional affect to the value of optimal performance. The author shows exemplary conditions for minimum specific fuel consumption that can be observed at an extremely high productivity and low productivity of the furnace.
Keywords: gas furnaces, heating the metal, performance, optimum mode.

\section{REFERENCES}

1. P anfjorov V.I. Izv. vuz. Chernaja metallurgija. 2007. № 10. pp. $53-56$.

2. Rozengart Ju.I., Potapov B.B., O1'shanskij V.M., B o rodulin A.V. Teploobmen i teplovye rezhimy v promyshlennyh pechah (Heat transfer and thermal rmodes of industrial furnaces). Kiev - Doneck: Vishha shkola, 1986. 296 p.

3. Sokolov A.K. Izv. AN. Jenergetika. 2007. № 2. pp. 126 - 136.

4. Revun M.P., Sok olov A.K. Adaptivnye sistemy upravlenija processami nagreva metalla: Monografija (Adaptive process control systems of metal heating: Monography). Zaporozh'e: Izd.-vo ZGIA, 1998. $351 \mathrm{p}$.

5. S o k olov A.K. Sovershenstvovanie i optimizacija nagreva metalla $v$ gazovyh pechah metodom matematicheskogo modelirovanija (Improvement and optimization of metal heating in gas furnaces by mathematical modeling). Ivanovo, 2012. $256 \mathrm{p}$.

6. S o k olov A.K. Izv. vuzov. Jenergetika. 1981. № 5. pp. $104-106$. Received 8 October 2013 